\title{
Movement characteristics and effects of GUI design on how older adults swipe in mid-air
}

Conference or Workshop Item

Accepted Version

Theil Cabreira, A. and Hwang, F. (2018) Movement characteristics and effects of GUI design on how older adults swipe in mid-air. In: ASSETS '18: 20th International ACM SIGACCESS Conference on Computers and Accessibility, 2224 Oct 2018, Galway, Ireland, pp. 420-422. (ISBN:

9781450356503) Available at http://centaur.reading.ac.uk/78995/

It is advisable to refer to the publisher's version if you intend to cite from the work. See Guidance on citing.

Published version at: https://doi.org/10.1145/3234695.3241014

Publisher statement: Permission to make digital or hard copies of part or all of this work for personal or classroom use is granted without fee provided that copies are not made or distributed for profit or commercial advantage and that copies bear this notice and the full citation on the first page. Copyrights for third- 
party components of this work must be honored. For all other uses, contact the Owner/Author.

All outputs in CentAUR are protected by Intellectual Property Rights law, including copyright law. Copyright and IPR is retained by the creators or other copyright holders. Terms and conditions for use of this material are defined in the End User Agreement.

\section{www.reading.ac.uk/centaur}

\section{CentAUR}

Central Archive at the University of Reading

Reading's research outputs online 


\section{Movement Characteristics and Effects of GUI Design on How Older Adults Swipe in Mid-Air}

\author{
Arthur Theil Cabreira \\ University of Reading \\ Reading, UK \\ a.theilcabreira@pgr.reading.ac.uk
}

\author{
Faustina Hwang \\ University of Reading \\ Reading, UK \\ f.hwang@ reading.ac.uk
}
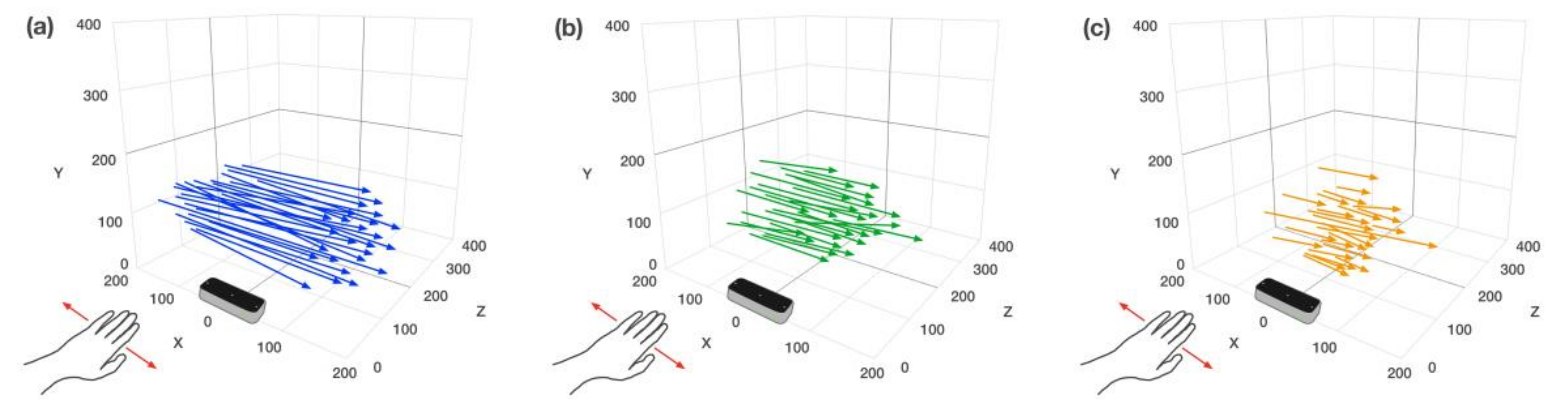

Figure 1. Swipe start and end positions (left to right) in mid-air for (a) large, (b) medium, and (c) small on-screen carousel-style menus. Arrows represent an average of 10 swipes per participant. Units are in $\mathbf{m m}$ within the Leap Motion's coordinate frame.

\begin{abstract}
We conducted a study with 25 older adults that aimed to investigate how older users interact with swipe-based interactions in mid-air and how menu sizes may affect swipe characteristics. Our findings suggest that currentlyimplemented motion-based interaction parameters may not be very well-aligned with the expectations and physical abilities of the older population. In addition, we find that GUI design can shape how older users produce a swipe gesture in mid-air, and that appropriate GUI design can lead to higher success rates for users with little familiarity with this novel input method.
\end{abstract}

Author Keywords

aging; Leap Motion; gesture-based interaction; motion sensors; user-defined gestures; freehand, in-air gestures

\section{ACM Classification Keywords}

H.5.2 [Information interfaces and presentation]: User interfaces - input devices \& strategies.

\section{INTRODUCTION AND BACKGROUND}

Motion-based interaction through mid-air gestures has become increasingly popular with the advancement of

Permission to make digital or hard copies of part or all of this work for personal or classroom use is granted without fee provided that copies are not made or distributed for profit or commercial advantage and that copies bear this notice and the full citation on the first page. Copyrights for thirdparty components of this work must be honored. For all other uses, contact the Owner/Author.

ASSETS '18, October 22-24, 2018, Galway, Ireland (C) 2018 Copyright is held by the owner/author(s). ACM ISBN 978-1-4503-5650-3/18/10.

https://doi.org/10.1145/3234695.3241014 motion sensors (e.g. Microsoft HoloLens, Leap Motion) and has been employed in a diverse range of applications such as interactive TV, vehicles, and public displays [9, 10, 13]. Research [4, 11] has found that older adults (aged 60 and older) may face greater challenges when interacting through mid-air gestures due to age-related decline in motor control, limited range of motion [5] and a lack of familiarity with this novel input method $[6,8,12]$, however this issue is far from being tackled and fully understood. A particular challenge relates to the difficulty and inconsistency of how older adults perform the swipe gesture, a highly recurrent mid-air gesture that involves a lateral swiping motion of a finger or hand, that is mostly used for menu navigation and item selection $[3,15,16]$. To date, there are limited insights on how older users interact with mid-air gesture interaction and whether gesture-based systems take into account how older users gesture in mid-air. Consequently, the growing older population could be excluded from emerging interfaces that are employing mid-air gestures in different interaction contexts. One method yet to be explored for minimising this problem is observing how older users intuitively swipe in mid-air in order to elicit movement data and improve sensing parameters. We report a study with 25 older adults that aimed to investigate how older users interact with swipe-based interactions in mid-air and how well the Leap Motion sensor is able to recognise those movements. Three on-screen carousel menu sizes were studied in our experimental design in order to investigate the possible effects of menu size on swipe characteristics. Our findings may contribute to the design and development of more inclusive sensing parameters and age-friendly gesture-based interfaces. 


\section{EXPERIMENT}

\section{Participants}

25 older adults (12 female) participated in the experiment. Participants were aged 60 to 83 (mean age: 67; SD=6.7) and had prior computer experience and some familiarity with touchscreen interaction. No prior experience with motion sensing devices and mid-air gesture interaction was reported. Before the start of the experiment, participants had their manual dexterity and motor skills assessed using a Rolyan 9-Hole Peg Toolkit [17], which confirmed that all participants were within the norms for their age group.

\section{Procedure and Apparatus}

Participants were first introduced to the Leap Motion sensor. They were then asked to complete a task that involved navigating through a 10-item carousel menu by swiping their hand left and right in mid-air (Figure 2). This task was repeated for three different carousel sizes which were - in pixels - 2130x560 (large), 1800x480 (medium), and 1390x330 (small). Participants were encouraged to swipe intuitively and were not given specific demonstrations on how to gesture. The carousel moved to the next item independently from how participants swiped, in order to encourage participants to swipe in a manner that was natural to them without trying to conform to a gesture recogniser. Movement data was being automatically logged for later analysis. The order of presentation of menu sizes was counterbalanced across participants. The session was video recorded. The carousel was developed using JavaScript [1] and movement data was collected using the Leap Motion sensor [16]. Participants interacted in a sitting position with a Leap Motion sensor connected to a 13-inch MacBook with built-in retina display at 2560x1600 pixels resolution (227 ppi).

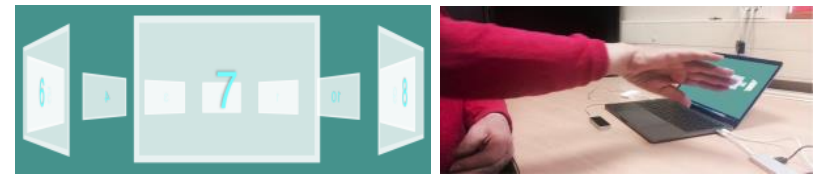

Figure 2. Carousel navigation and experimental set-up.

\section{RESULTS}

Table 1 shows average swipe characteristics for each carousel menu size. 395 out of 750 swipe trials $(52.7 \%)$ were successfully recognised by the Leap Motion sensor.

\begin{tabular}{llll}
$\begin{array}{l}\text { Carousel } \\
\text { menu size } \\
\text { (pixels) }\end{array}$ & $\begin{array}{l}\text { Swipe } \\
\text { recognition } \\
\text { rate }\end{array}$ & $\begin{array}{l}\text { Average } \\
\text { swipe length } \\
(\mathbf{m m})\end{array}$ & $\begin{array}{l}\text { Average } \\
\text { swipe speed } \\
(\mathbf{m m} / \mathbf{s})\end{array}$ \\
\hline $\begin{array}{l}\text { Large } \\
2130 \times 560\end{array}$ & $90 \%$ & $\begin{array}{l}210.9 \\
(\mathrm{SD}=35.8)\end{array}$ & $\begin{array}{l}889.1 \\
(\mathrm{SD}=57.8)\end{array}$ \\
\hline $\begin{array}{l}\text { Medium } \\
1800 \times 480\end{array}$ & $50 \%$ & 134.1 & 482.7 \\
\hline Small & & $(\mathrm{SD}=50.4)$ & $(\mathrm{SD}=61.5)$ \\
$1390 \times 330$ & $18 \%$ & 97.8 & 291.5 \\
\hline
\end{tabular}

Table 1. Movement characteristics of the swipe gesture made in mid-air by older adults for three carousel menu sizes.

Two participants chose to swipe using the index finger, whilst the other 23 participants swiped using the entire hand. Figure 1 shows, for each participant, the average starting and ending positions for their swipes, relative to the sensor for each menu size. A repeated-measures MANOVA showed a significant main effect of carousel menu size on how older adults swipe in mid-air $[\mathrm{F}(2,392)=56.5$; $\mathrm{p}$ $=.0002]$. A Tukey HSD post-hoc analysis found that carousel menu size had an effect on all dependent variables (swipe recognition rate, length, and speed). That is, the swiping motion was significantly longer $(\mathrm{p}=.0015)$, faster $(\mathrm{p}=.01)$, and better recognised $(\mathrm{p}=.01)$ when older adults were navigating through the larger carousel menu. In the same way, length, speed and recognition rates significantly decreased when interacting with the medium and small carousel menus respectively.

\section{DISCUSSION AND CONCLUSION}

The Leap Motion default parameters for a swipe gesture include a minimum swipe speed of $1000 \mathrm{~mm} / \mathrm{s}$ and a minimum swipe length of $150 \mathrm{~mm}$ [15]. However, the results from the current study suggest that those parameters may not be age-friendly and may not have taken into consideration the psychomotor aspects of how older adults naturally swipe in mid-air. This mismatch between how older adults naturally swipe and how the system expects them to do it is likely to lead to a number of failed interaction attempts (see recognition rates in Table 1) and may affect the overall usability and accessibility of gesturebased interfaces. Furthermore, we also found that carousel menu size affected how older adults produced a mid-air swipe gesture to a point where it was almost unrecognisable by the sensor. The large carousel menu (2130x560 pixels) was the menu with highest recognition rate $(90 \%)$, whilst the smallest menu size (1390x330 pixels) achieved only $18 \%$ due to swipe lengths and speeds so low that the sensor was incapable of interpreting the motions as a recognised swipe. Our findings emphasise the role of GUI design in gesture-based systems, and how user interface choices can not only shape how older users produce a gesture in mid-air but also lead to higher success rates for users with little familiarity with this novel input method. Our findings suggest that currently-implemented motion-based interaction parameters may not be necessarily aligned with the expectations and physical abilities of the older population and we expect that our work will contribute to the implementation of gesturing parameters that take older users into account, leading to a higher success rate and greater accessibility for gesture-based interfaces.

\section{ACKNOWLEDGMENTS}

We thank all the volunteers for their participation and the University of Reading's Hugh Sinclair Unit for their help with recruitment. The research is funded by the Brazilian Science Without Borders Programme, an initiative under the Ministry of Education and CAPES Foundation. Project number BEX 13037-13-7.

\section{REFERENCES}

1. 3D Carousel Using TweenMax.js \& jQuery. Available at: https://codepen.io/johnblazek/pen/nceyw 
2. Anna Dickinson, Roos Eisma, and Peter Gregor. 2011. The barriers that older novices encounter to computer use. Universal Access in the Information Society. 10, 3, 261-266. https://doi.org/10.1007/s10209-010-0208-6

3. Arthur Theil Cabreira and Faustina Hwang. 2015. An analysis of mid-air gestures used across three platforms. In Proceedings of the 2015 British HCI Conference (British HCI '15), 257-258. http://dx.doi.org/10.1145/2783446.2783599

4. Arthur Theil Cabreira and Faustina Hwang. 2016. How Do Novice Older Users Evaluate and Perform Mid-Air Gesture Interaction for the First Time?. In Proceedings of the 9th Nordic Conference on Human-Computer Interaction (NordiCHI '16), 122-128. https://doi.org/10.1145/2971485.2996757

5. Caroline J. Ketcham and George E. Stelmach. 2001. Age-Related Declines in Motor Control. In Birren, J. E. \& Schaie, K.W. (Eds.), Handbook of the Psychology of Aging. Academic Press.

6. Christian Stößel. 2009. Familiarity as a factor in designing finger gestures for elderly users.

In Proceedings of the 11th International Conference on Human-Computer Interaction with Mobile Devices and Services (MobileHCI '09), Article 78. http://dx.doi.org/10.1145/1613858.1613950

7. Darren Guinness, G. Michael Poor, and Alvin Jude. 2014. Gestures with speech for hand-impaired persons. In Proceedings of the 16th international ACM SIGACCESS conference on Computers \& accessibility (ASSETS '14). ACM, New York, NY, USA, 259-260.

http://dx.doi.org/10.1145/2661334.2661398

8. Donald A. Norman and Jakob Nielsen. 2010. Gestural interfaces: a step backward in usability. interactions 17, 5 (September 2010), 46-49. https://doi.org/10.1145/1836216.1836228

9. Gözel Shakeri, John H. Williamson, and Stephen Brewster. 2017. Novel Multimodal Feedback Techniques for In-Car Mid-Air Gesture Interaction. In Proceedings of the 9th International Conference on Automotive User Interfaces and Interactive Vehicular Applications (AutomotiveUI '17). ACM, New York, NY, USA, 84-93. https://doi.org/10.1145/3122986.3123011

10. Jan Bobeth, Susanne Schmehl, Ernst Kruijff, Stephanie Deutsch, and Manfred Tscheligi. 2012. Evaluating performance and acceptance of older adults using freehand gestures for TV menu control. In Proceedings of the 10th European Conference on Interactive TV and Video (EuroITV '12). ACM, New York, NY, USA, 35-44. https://doi.org/10.1145/2325616.2325625

11. Kathrin Gerling, Ian Livingston, Lennart Nacke, Regan Mandryk. 2012. Full-body motion-based game interaction for older adults. In Proceedings of the
SIGCHI Conference on Human Factors in Computing Systems (CHI '12), 1873-1882.

http://dx.doi.org/10.1145/2207676.2208324

12. Leah Findlater, Jon E. Froehlich, Kays Fattal, Jacob O. Wobbrock, and Tanya Dastyar. 2013. Age-related differences in performance with touchscreens compared to traditional mouse input. In Proceedings of the SIGCHI Conference on Human Factors in Computing Systems (CHI '13). ACM, New York, NY, USA, 343-346. DOI:

https://doi.org/10.1145/2470654.2470703

13. Robert Walter, Gilles Bailly, Nina Valkanova, and Jörg Müller. 2014. Cuenesics: using mid-air gestures to select items on interactive public displays.

In Proceedings of the 16th international conference on Human-computer interaction with mobile devices \& services (MobileHCI '14). ACM, New York, NY, USA, 299-308. https://doi.org/10.1145/2628363.2628368

14. Sara J. Czaja and Chin Chin Lee. 2007. Information technology and older adults. In J. A. Jacko \& A. Sears (Eds.), The Human Computer-Interaction Handbook (2nd ed., 777-792. Lawrence Erlbaum Associates, New York.

15. Swipe Gesture - Leap Motion Documentation. Available at: https://developerarchive.leapmotion.com/documentation/v2/python/api/ Leap.SwipeGesture.html?proglang=python

16. Wei Lu, Zheng Tong, Jinghui Chu. 2016. Dynamic Hand Gesture Recognition With Leap Motion Controller. IEEE Signal Processing Letters. 23, 9: 1188-1192.

17. Ying Chih Wang, Richard W. Bohannon, Jay Kapellusch, Arun Garg, Richard C. Gershon. 2015. Dexterity as measured with the 9-Hole Peg Test (9HPT) across the age span. Journal of Hand Therapy. 28, 1, 53-60. 